\title{
Transgender Health Care in the U.S. Military and Veterans Health Administration Facilities
}

\author{
Michael R. Kauth ${ }^{1,2,3,4,5}$ - John R. Blosnich ${ }^{6,7} \cdot$ Janelle Marra $^{8}$ - Zander Keig ${ }^{8}$. \\ Jillian C. Shipherd ${ }^{2,9,10,11}$
}

Published online: 27 July 2017

(C) The Author(s) 2017. This article is an open access publication

\begin{abstract}
Purpose of the Review Health care for transgender veterans in the United States (U.S.) Veterans Health Administration (VHA) is relatively new and for active duty service members (ADSMs) in the military is quite recent. Prevalence of transgender veterans and ADSMs, health conditions, and healthcare provision in VHA and military facilities are reviewed.

Recent Findings There are approximately 134,300 transgender veterans and 15,000 ADSMs. Based on diagnostic codes, more than 5000 transgender veterans receive care in VHA. Transgender veterans experience higher rates of most mental and physical health conditions compared to non-transgender veterans. Comprehensive health care is provided at VHA facilities, except surgical interventions for gender confirmation, and is beginning to be provided in military facilities.

Summary While VHA and military facilities have increased access to health care for transgender veterans and ADSMs,
\end{abstract}

This article is part of the Topical Collection on Variations in Orientation, Identity, Addiction, and Compulsion

Michael R. Kauth

Michael.Kauth@va.gov

1 Michael E. DeBakey VA Medical Center, 2002 Holcombe Blvd, ATTN: VA 152, Houston, TX 77030, USA

2 Lesbian, Gay, Bisexual, and Transgender (LGBT) Health Program, Veterans Health Administration, Washington, DC 20571, USA

3 VA South Central Mental Illness Research, Education, and Clinical Center, Houston, TX 77030, USA

4 Houston VA Health Services Research \& Development Center for Innovations in Quality, Effectiveness and Safety,

Houston, TX 77030, USA determining outcomes of care is premature. Healthcare delivery efforts alone are unlikely to erase health disparities experienced by this group.

Keywords Transgender $\cdot$ Veteran $\cdot$ Military $\cdot$ Healthcare $\cdot$ Active duty

\section{Introduction}

Until recently, individuals with "transgender conditions" were disqualified from joining or serving in the United States (U.S.) military [1]. In July 2015, Secretary of Defense Ashton Carter charged a workgroup to study the policy and readiness implications of allowing transgender persons to serve openly [2]. At the same time, Carter allowed for a relaxation of the policy to discharge current transgender military personnel. In
5 Baylor College of Medicine, Houston, TX 77030, USA

6 VA Pittsburgh Healthcare System, Center for Health Equity Research and Promotion, Pittsburgh, PA 15260, USA

7 Division of General Internal Medicine, School of Medicine, University of Pittsburgh, Pittsburgh, PA 15260, USA

8 Naval Medical Center San Diego, San Diego, CA 92134, USA

9 National Center for PTSD, Women's Health Sciences Division, Boston, MA 02130, USA

10 VA Boston Healthcare System, Boston, MA 02130, USA

11 Boston University School of Medicine, Boston, MA 02130, USA 
June 2016, the U.S. Department of Defense (DoD) announced a change in policy permitting transgender persons to serve openly and by extension receive transition-related health care [1]. Currently, 19 countries including the U.S. allow transgender persons to serve openly in the military [3].

Despite the ban on open service, it was estimated in 2014 that 15,500 transgender individuals were on active duty or in the National Guard or Reserves and 134,300 transgender persons had prior military service or were in the Guard/Reserves (i.e., veterans) (approximately $0.6 \%$ of total service members) $[3,4]$. Applying prevalence rates to military data, others have estimated that 2450 transgender service members are on active duty and 1510 are in the National Guard or Reserves (accounting for $0.1-0.5 \%$ of total service members) [2].

U.S. veterans who have not been dishonorably discharged may be eligible for and seek health care at the Veterans Health Administration (VHA), comprised of 1233 healthcare facilities and annually treating nearly 9 million of the approximately 22 million veterans $[5,6]$. The VHA has never prohibited general healthcare services for transgender veterans and, in 2011 , issued a national directive mandating transition-related services such as hormone therapy but not surgical interventions, which are currently prohibited by VHA regulation [7]. Although VHA does not currently track self-identified gender identity, Blosnich and colleagues were able to identify veterans who sought transgender-related care using two ICD-9 diagnostic codes associated with the visit [8]. Using these methods and VHA medical records from 2000 to 2011, the investigators estimated that prevalence rates for transgender veterans in VHA (22.9/100,000 persons) are over five times higher than in the general U.S. population $(4.3 / 100,000$ persons) [8].

Since the release of the VHA directive permitting transgender individuals to serve openly, there has been an increase in the use of transgender-related diagnostic codes [9]. Using four ICD-9 diagnostic codes (gender identity disorder, gender identity disorder not otherwise specified, transsexualism, and transvestic fetishism), Brown and Jones identified 5135 unique transgender veterans in VHA between 1996 and $2013[10 \bullet \cdot$. Using the same codes but different dates (1997-2014), other investigators identified 6308 transgender veterans receiving care in VHA [11]. Diagnostically derived data likely underestimate the population of transgender veterans in VHA because some veterans may not identify themselves as transgender to their providers, including some who get their transition-related care outside of VHA.

This paper reviews the recent literature on health conditions experienced by transgender veterans and describes healthcare provision for this population in VHA and military facilities. It should be noted that the paucity of studies of active duty service members (ADSMs) is due to the risks for discharge for service members who disclosed their transgender status under earlier regulations.

\section{Health Conditions Among Transgender Veterans}

Transgender veterans, both a stigmatized gender minority and a minority as veterans, may experience dual health disparities [12]. Growing evidence supports the view that the chronic stress and stigma associated with minority identities and experiences (e.g., transgender and veteran) -also known as minority stress - contribute to poorer health compared with nontransgender people and non-veterans [13, 14]. Indeed, in a study of VHA veterans in states where non-discrimination policies in employment included gender identity, transgender veterans living in states with these protections had fewer mental health diagnoses than transgender veterans living in states without protections [11]. Additionally, from VHA medical record data, individual-level indicators of social stresshomelessness, financial strain, and violence-were strongly associated with physical and mental health diagnoses among transgender veterans [11].

Further evidence underscoring the effects of minority stress was found in a separate study looking at racial health disparities in a VHA sample of 5135 transgender veterans identified using ICD-9 diagnostic codes. Investigators found that black transgender veterans had increased odds of being diagnosed with alcohol abuse, benign prostatic hyperplasia, congestive heart failure, HIV, hypertension, renal disease, serious mental illness, and tobacco use relative to white transgender veterans [15••]. However, black transgender veterans were less likely to be diagnosed with depression, hypercholesterolemia, and obesity than white transgender veterans. Nevertheless, black transgender veterans had higher rates of homelessness and incarceration suggesting overall greater social disadvantages and more medical and mental health conditions compared to white transgender veterans.

Studies using non-VHA data have found similar indicators of minority stress. A qualitative analysis of responses from a national convenience survey of 201 transgender veterans (68\% transgender women; $87 \%$ white; ages $18-83$, mean age $=49.9$ years) identified common stressors that likely affect health [16]. These stressors included external minority stress (e.g., failed marriage due to spouse's non-acceptance), internal minority stress (e.g., non-disclosure, fear of rejection), VHA services (e.g., lack of gender confirming surgeries, being misgendered), military experience (e.g., not able to be open, forced discharge), general health care experience (e.g., educating providers), and lack of community (e.g., not accepted). In another publication from this sample, $34 \%$ of respondents reported experiencing employment discrimination and homelessness [17]. Given the stigma and discrimination faced by transgender veterans, deleterious effects on mental health outcomes are not surprising.

In the online survey of 201 transgender veterans described above, more than half (57\%) of respondents reported past-year suicidal ideation and $66 \%$ noted a history of suicide plans or 
attempts [17]. Several factors were positively associated with suicidal ideation and attempts, including older age, transgender male identity, felt stigma associated with gender identity during the military, history of homelessness, and current traumatic stress and depressive symptoms. Using VHA data, Blosnich and colleagues found that rates of non-fatal suicide-related events (e.g., voiced plans, attempts) among transgender veterans (with relevant ICD-9 codes) were 20 times higher than for the general VHA population [8]. The crude suicide rate among transgender veterans in VHA (between 2000 and 2009) was 82/100,000 person-years, which is higher than the general VHA population rate but similar to crude suicide rates for VHA veterans with serious mental illnesses [18]. The average age of suicide deaths among transgender veterans in VHA was 49.4 years, while the average age of suicide deaths for non-transgender VHA veterans was between 54.5 and 59.6 years [19], suggesting that transgender veterans experience more years of potential life lost.

Using ICD-9 codes, Lindsay and colleagues identified 332 transgender veterans (78 men, 254 women; 73\% white; mean age $=33.86$ years) who had served in recent conflicts in Iraq and Afghanistan [20]. There were few significant differences between transgender men and women but, overall, the sample exhibited high rates of mental health diagnoses: $70 \%$ had a depressive disorder, 52\% an anxiety disorder, and 52\% were diagnosed with post-traumatic stress disorder (PTSD). In addition, $15 \%$ of the sample had experienced a military sexual trauma, which can include sexual assault or repeated, threatening sexual harassment during military service [21].

In a matched case-control sample of 5135 transgender veterans (69\% male assigned at birth; $80 \%$ white; mean age $=55.8$ years) in VHA (between 1996 and 2013), transgender veterans were significantly more likely to have experienced military sexual trauma (15 vs. 6\%), been in combat (13 vs. $11 \%$ ), and have a service-connected disability (47 vs. $42 \%$ ) compared to the control group of VHA-using cisgender veterans $[10 \bullet \bullet$. Transgender veterans were significantly more likely to have all ten mental health conditions examined, including depression, suicidality, serious mental illness, PTSD, and alcohol abuse. Further, transgender veterans had significantly higher rates of all medical conditions examined, with the exception of breast cancer and cirrhosis. Transgender veterans in VHA had higher rates of heart disease, pulmonary disease, hypertension, diabetes, hypercholesterolemia, and renal disease compared to non-transgender veterans. One of the largest discrepancies was in rates of HIV, with the odds of HIV seropositivity nearly five times greater for transgender veterans compared to controls. Lastly, transgender veterans compared to cisgender veterans had higher rates of homelessness (30 vs. $10 \%$ ) and incarceration (3 vs. 1\%) [10••].

Lastly, Hoy-Ellis and colleagues examined a subsample of 183 older transgender adults (50 or older) who participated in the 2014 Aging with Pride: National Health, Aging, and
Sexuality Study, a national longitudinal survey of 2450 lesbian, gay, bisexual, and transgender (LGBT) older adults [22]. More than $60 \%$ were white, nearly half of the subsample identified as women, $28 \%$ as men, and $26 \%$ as another gender. Interestingly, $22 \%$ had military service $(n=43)$ and this predicted lower depressive symptoms and higher psychological health-related quality of life scores compared to those without military service. This study illustrates how participant recruitment to a study (e.g., medical records vs. survey) can influence findings; each type of sampling tells a different piece of the story. The authors hypothesized that military service for transgender persons may contribute to resilience and positive mental health outcomes.

\section{Care for ADSMs at Military Facilities}

Care for transgender ADSMs at military facilities began only in June 2016 when DoD policy changed to allow transgender individuals to serve openly in the military [1]. Each branch of the military has a separate healthcare policy for transgender ADSMs, which includes providing hormone therapy, primary care, mental health services, and gender confirmation surgeries. At present, availability of these services varies based on location, with significant limitations in many operational units and overseas locations. A summary of each branch's current policy and procedures follows below. Military readiness and gender-based uniform, housing, and performance standards are unique aspects of gender transition for ADSMs. Consequently, the ADSM's Commanding Officer must approve the individual's transition plan and initiation date, as well as any subsequent change in gender marker on official DoD records. ADSMs are recognized by the gender designated in their personnel record and are responsible for meeting all standards for uniforms, grooming, fitness standards, housing, bathroom, and shower facilities in accordance with that gender, until the gender marker is changed or they receive an exception to the policy. Exceptions to policy are made on a case-by-case basis, initiated by the ADSM, and approved by higher levels of administration in each service branch. The plan to allow individuals enlisting in the military who identify as transgender to have at least 18 months stability in their transitioned gender identity before entering service, beginning July 1, 2017, has been delayed six months [23].

The Navy Bureau of Medicine and Surgery established two regional transgender care teams (at Naval Medical Center at Portsmouth and Navy Medical Center at San Diego) staffed with an interdisciplinary team of medical, surgical, and behavioral health providers [24]. An ADSM who requests to initiate gender transition is evaluated by a mental health provider who establishes a diagnosis of Gender Dysphoria [25] and determines that transition is medically necessary. The ADSM is also evaluated by a medical provider to determine medical 
clearance for hormone therapy. Fertility counseling is conducted to discuss options for fertility preservation, consistent with Endocrine Society guidelines [26]. The case is referred to one of the regional transgender care teams to confirm the diagnosis (or request additional assessment) and create or validate the medical transition plan and completion date. The Transgender Care Team drafts a medical transition plan and uploads the plan in the ADSM's electronic health record along with a memorandum to the ADSM's Commanding Officer. Treatment modalities may include psychotherapy, psychopharmacology, recommendations to live in the new gender (a.k.a. real life experience), hormone therapy, and surgical interventions. The Commanding Officer has 90 working days to approve or defer the proposed treatment plan. Gender markers may be updated in the personnel record system when the Transgender Care Team determines that the ADSM's individualized transition plan is complete and the ADSM can meet the requirements of their new gender. Medical and surgical treatments may continue after completion of the care plan.

Similar to Navy policy and procedures, Air Force policy requires the ADSM to have a diagnosis of gender dysphoria and a statement of medical necessity for transition by a military healthcare provider [27]. The diagnosis is confirmed via chart review by the medical multidisciplinary team in San Antonio, TX. Recommendations from the medical provider and medical multidisciplinary team are used to develop an individualized treatment plan. Unit commanders have 90 days to approve or delay the treatment plan based on unit readiness. Unlike the Navy and Air Force policies, Army Directive 2016-35 decentralizes formation of the treatment plan [28]. The army policy requires the ADSM to receive a diagnosis of Gender Dysphoria from a military provider with a statement of medical necessity for transitioning. The medical provider formulates a treatment plan and estimated timeline for completion. The ADSM notifies the brigade level commander of the treatment plan and timeline who has 30 days to approve or delay the care plan.

DoD policy establishes that military medical and behavioral health providers will receive training in transgender care [1]. Training for military providers is still new and, like training in VHA, will likely be evolving in format and intensity. A survey of 180 military healthcare professionals attending the Uniformed Services Academy of Family Physicians, shortly before the transgender service ban was lifted, found that $37.3 \%$ of respondents had treated a transgender patient [29]. However, only $47.1 \%$ of respondents were willing to prescribe hormones to an eligible transgender patient. Those who were unwilling to prescribe hormones cited ethical concerns, discomfort, or both. The majority $(87.1 \%)$ of respondents felt they had insufficient training to prescribe hormones for transgender patients.
As more transgender ADSM receive care within military facilities, it is likely that current policy and practices will need to be amended and additional policies may be required. For example, the impact of gender transition on personnel in combat zones is uncertain. In addition, the military needs to formalize a similar interdisciplinary care process for both adult (e.g., spouses, retirees) and pediatric non-active duty patients (i.e., dependent children) who are eligible for TRICARE benefits.

\section{Clinical Care and Practices at VHA Facilities}

The 2011 transgender healthcare directive established a standard of care across the VHA system, which included gender counseling, hormone therapy, and post-operative care for medically necessary complications (e.g., infection, bleeding, constriction of the neovagina) but not gender confirming surgeries [7]. At present, VHA is prohibited by regulation from conducting or subsidizing gender confirming surgeries, although VHA leadership expressed support for removing this regulation in November 2016, while noting that funding for these procedures was not currently available [30].

By 2011, transgender veterans were becoming more visible [9], but few VHA clinicians had experience or training in transgender care. VHA developed levels of education and training materials for staff, including an internal SharePoint website repository for articles, clinical resources, information about support groups, LGBT health awareness materials, and links to policies and guidelines [31•]. In addition, subject matter experts created three web-based programs (with continuing medical education) on transgender veteran health care, now available to the public through VHA TRAIN (https://vha.train. org/), a free service of the Public Health Foundation. The first program is a general introduction to transgender health topics for clinical and non-clinical staff. The second program teaches mental health providers how to make an appropriate diagnosis and conduct an evaluation for hormone therapy. The third program provides guidance for prescribing hormone therapy and managing other medical conditions. These early national training efforts and the need for policy leaders contributed to the formation of the LGBT Health Program within the VHA Office of Patient Care Services in 2012, with subsequent training initiatives led through this program office [31•].

In addition, VHA established a national e-consultation service on transgender care. Any provider can submit clinical questions through the patient's chart and receive a quick response from a multidisciplinary team experienced in transgender care [32]. Since its launch in mid-2014, the transgender econsultation service has received more than 700 consults for 554 unique veterans. VHA also implemented an intensive training program for interdisciplinary teams of providers through videoconferencing to develop experienced teams 
throughout the system [33, 34]. Learning teams were comprised of at least one behavioral health provider and one medical provider, although most teams have averaged five members. Learning teams joined one-hour, twice-monthly sessions via videoconferencing for 7 months. Each session consisted of a brief $(20 \mathrm{~min})$ didactic on transgender care and then casebased consultation. Each team presented at least one case, while other teams listened and asked questions. To date, this program has trained 75 teams and nearly 600 clinical providers, including 3 teams of military providers. In addition, VHA through the Offices of Academic Affiliations and Mental Health created nine one-year postdoctoral psychology fellowships in LGBT health, now in their fourth year (for a list of sites go to: www.patientcare.va.gov/LGBT/LGBT Veteran_Training.asp). These LGBT health fellows provide direct clinical services to transgender veterans, train staff, and outreach to LGBT community organizations. Recent LGBT health fellows have rated their training positively, and more than half $(56 \%)$ have accepted staff positions in the VHA or other federal agencies [35].

Access to Care All VHA patients have a primary care provider, but veterans can be reluctant to raise gender identity questions with primary care providers [36]. Veterans who are in treatment with a mental health provider may find it easier to begin discussions about accessing transition-related care [37]. However, for veterans who want to know what services are available to them without disclosing information to a provider, having an ally in the system for general questions can be helpful. In 2016, VHA asked each healthcare facility to appoint an LGBT Veteran Care Coordinator (VCC) to assess the need for and expand clinical services, train staff, and support the facility in creating a welcoming environment for LGBT veterans. Each facility protects some percentage of time for the LGBT VCC to perform their duties, and some facilities have appointed more than one LGBT VCC. To date, 175 LGBT VCCs serve the 168 VHA medical centers. These staff can be a helpful resource to inform veterans about what services are available to them and to serve as an ally to assist when questions or concerns arise.

Outcomes Despite these numerous initiatives, scant evidence of their effect on clinical care or health outcomes for transgender veterans exists. This research has not been done. In part, these initiatives are new and insufficient time has passed to demonstrate substantive effects on patient care. However, VHA efforts to provide equitable, quality care for transgender veterans are unlikely to mitigate decades of health disparities rooted in societal stigma and prejudice.

Nevertheless, the VHA has significantly improved access to quality care for transgender veterans through training and awareness efforts, notably the more than 75 interdisciplinary teams of providers participating in intensive case-based teleconferencing training and 517 providers who have received e-consultation services. Through these efforts VHA has increased both the number of providers offering care to transgender veterans and providers' underlying understanding of quality transgender care. The LGBT VCCs also function to fill gaps in needed local clinical services and serve as a safety net to ensure continuity of care. The increasing number of transgender veterans identified in VHA suggests that education and training efforts are working [9].

Another suggestive outcome of efforts to create a more welcoming environment is the number of VHA facilities participating in and achieving leadership status with the Human Rights Campaign's Healthcare Equality Index (HEI; http:// www.hrc.org/hei). The HEI is a voluntary assessment of healthcare agencies' responsiveness to LGBT patients and employees they serve across several domains, including non-discrimination policies, staff training, information provided to patients, and electronic records. Results of the 2015 HEI survey demonstrated that $84 \%$ (96 of 114) of participating VHA facilities achieved leadership status [38]. However, the 2016 HEI results showed only $45 \%$ (45 of 101) of VHA facilities obtained leadership status, in part because the HEI scoring criteria became more stringent [39] and in part because the VHA did not encourage participation as strongly as it had in previous years. While there is room for improvement, these successes reflect national and local efforts to create LGBT-friendly clinical environments.

Next Steps Though existing research highlights several areas of need in transgender health and health care, transgender veteran and ADSM health care is an emerging field. Generally, recommendations around transgender health for veterans and ADSMs can be envisioned in at least two levels. First, studies of health system-level factors for transgender health are critically important and include several forms of research. For example, VHA and DoD should evaluate their training efforts to identify facilitators and barriers around providers implementing their training to deliver appropriate care to transgender patients. Additionally, epidemiologic research would benefit greatly from standardized collection of selfidentified gender identity in electronic health record data [40 $]$. Finally, assessments are needed of patient outcomes and satisfaction with care from providers who have received training.

Correspondingly, individual-level studies are needed as changes are made to the health system. Improved healthcare practices for transgender veterans may lead to greater patient satisfaction, which requires inclusion of gender identity data in patient experience surveys. Research should also include development and evaluation of tailored individual-level therapies, investigating the efficacy either of proven treatments (e.g., the application of cognitive behavioral therapy to reduce suicide risk among 
transgender patients) or of specific modalities tailored to transgender populations (e.g., PTSD support groups specifically for transgender patients).

Investigators should also engage transgender stakeholders in the research process. Inclusion of transgender individuals in the research planning process not only provides the opportunity to design culturally informed and respectful research, it also enhances the ability of historically underserved and stigmatized populations to reclaim agency in research about their health.

\section{Conclusions}

The DoD has just begun providing health care to transgender ADSMs. Although the VHA has provided care to transgender veterans for decades, a national directive mandating standards of care, formal provider training and clinical support, and efforts to create more welcoming clinical environments are relatively recent (since 2011). Some evidence suggests that VHA has improved access to equitable, quality care for transgender veterans, but studies about improved health outcomes are needed. It is possible that VHA and DoD efforts may improve individual patient care but not fully mitigate health disparities experienced by transgender veterans and ADSMs. It is also possible that societal change may contribute to reductions in the stigma and prejudice transgender people face, likely benefitting their access to quality health care, improving self-care and self-esteem, and thereby reducing health disparities.

Acknowledgements This work was supported partially by a career development award to JRB from the US Department of Veterans Affairs, Health Services Research \& Development (CDA 14-408). This work was also supported by funding to MRK from the VA South Central Mental Illness Research, Education and Clinical Center and resources from the Houston VA HSR\&D Center of Innovations of Quality, Effectiveness and Safety (CIN 13-413) at the Michael E. DeBakey VA Medical Center. The opinions expressed in this work are those of the authors and do not necessarily represent those of the funders, institutions, the Department of Veterans Affairs, or the U.S. Government.

\section{Compliance with Ethical Standards}

Conflict of Interest Work by all authors was completed as part of their employment by the U.S. Government.

Human and Animal Rights and Informed Consent This article contains no studies with human or animal subjects performed by any of the authors.

Open Access This article is distributed under the terms of the Creative Commons Attribution 4.0 International License (http:// creativecommons.org/licenses/by/4.0/), which permits unrestricted use, distribution, and reproduction in any medium, provided you give appropriate credit to the original author(s) and the source, provide a link to the Creative Commons license, and indicate if changes were made.

\section{References}

Papers of particular interest, published recently, have been highlighted as:

- Of importance

- Of major importance

1. DoD Instruction (DoDI) 1300.28: In-service transition for service members identifying as transgender, June 30, 2016. Retrieved from https://www.defense.gov/Portals/1/features/2016/0616_policy/ DoD-Instruction-1300.28.pdf.

2. Schaefer AG, Iyengar R, Kadiyala S, Kavanagh J, Engel CC, Williams KM, Kress AM. Assessing the implications of allowing transgender personnel to serve openly. RAND National Defense Research Institute 2016. Retrieved from http://www.rand.org/ pubs/research reports/RR1530.html.

3. Elders J, Steinman AM: Report of the Transgender Military Service Commission. The PALM Center, San Francisco, CA. March 2014. Retrieved from http://archive.palmcenter.org/files/Transgender\% 20Military\%20Service\%20Report_0.pdf.

4. Gates GJ, Herman JL: Transgender military service in the United States. The Williams Institute, University of California-Los Angeles. May 2014. Retrieved from http://escholarship.org/uc/ item/1t24j53h\#page-1.

5. Veterans Health Administration. About VHA. 2017. Retrieved from https://www.va.gov/health/aboutvha.asp.

6. National Center for Veterans Analysis and Statistics. Veteran population. 2017. Retrieved from https:/www.va.gov/vetdata/veteran population.asp.

7. Veterans Health Administration Directive 2011-024: Providing health care to transgender and intersex veterans. Now VHA Directive 2013-003: Providing health care to transgender and intersex veterans. Retrieved from www.va.gov/vhapublications/ ViewPublication.asp?pub ID=2863.

8. Blosnich JR, Brown GR, Shipherd JC, Kauth MR, Piegari R, Bossarte RM. Prevalence of gender identity disorder and suicide risk among transgender veterans utilizing Veterans Health Administration care. Am J Pub Health. 2013;103(10):e27-32 doi:10.2105/AJPH.2013.301507.

9. Kauth MR, Shipherd JC, Lindsay J, Blosnich JR, Brown GR, Jones KT. Access to care for transgender veterans in the Veterans Health Administration: 2006-2013. Am J Pub Health. 2014;104(S4):e1-3. doi:10.2105/AJPH.2014.302086.

10.• Brown GR, Jones KT. Mental health and medical health disparities in 5135 transgender veterans receiving healthcare in the Veterans Health Administration: a case-control study. LGBT Health. 2016;3(2):122-31. doi:10.1089/lgbt.2015.0058. This casecontrol study identifies major health differences between transgender and non-transgender veterans in VHA.

11. Blosnich JR, Marsiglio MC, Dichter M, Gao S, Gordon AJ, Shipherd JC, et al. Impact of social determinants of health on medical conditions among transgender veterans. Am J Prev Med. 2017;52(4):491-8. doi:10.1016/j.amepre.2016.12.019.

12. Mattocks KM, Kauth MR, Sandfort T, Matza AR, Sullivan JC, Shipherd JC. Understanding health-care needs of sexual and gender minority veterans: how targeted research and policy can improve health. LGBT Health. 2014;1(1):50-7. doi:10.1089/lgbt.2013.0003.

13. Hendricks ML, Testa RJ. A conceptual framework for clinical work with transgender and gender nonconforming clients: an adaptation of the minority stress model. Prof Psych Res Pr. 2012;43(5):460. doi:10.1037/a0029597.

14. Meyer IH. Prejudice as stress: conceptual and measurement problems. Am J Pub Health. 2003;93(2):262-5. doi:10.2105/ AJPH.93.2.262. 
15.• Brown GR, Jones KT. Racial health disparities in a cohort of 5,135 transgender veterans. J Racial and Ethnic Health Disparities. 2014;1(4):257-66. doi:10.1007/s40615-014-0032-4. Consistent with minority stress theory, this study shows that transgender veterans of color have worse health outcomes than white transgender veterans.

16. Chen JA, Granato H, Shipherd JC, Simpson T, Lehavot K. A qualitative analysis of transgender veterans' lived experiences. Psychol Sex Orientat Gend Divers. 2017;4(1):63-74. doi:10.1037/ sgd0000217.

17. Lehavot K, Simpson TL, Shipherd JC. Factors associated with suicidality among a national sample of transgender veterans. Suicide Life Threat Behav. 2016;46(5):507-24. doi:10.1111/sltb. 12233.

18. Blosnich JB, Brown GR, Wojcio S, Jones KT, Bossarte RM. Mortality among veterans with transgender-related diagnoses in the Veteran Health Administration, FY2000-2009. LGBT Health. 2014;1(4):269-76. doi:10.1089/lgbt.2014.0050.

19. Kemp J, Bossarte R. Suicide data report, 2012. Department of Veterans Affairs. Retrieved from http://wiiwarrior.org/downloads/ suicide-data-report-2012-final.pdf.

20. Lindsay JA, Keo-Meier C, Hudson S, Walder A, Martin LA, Kauth MR. Mental health of transgender veterans of the Iraq and Afghanistan conflicts who experienced military sexual trauma. J Traumatic Stress. 2016;29(6):563-367. doi:10.1002/jts.22146.

21. U.S. Department of Veterans Affairs: PTSD: National Center for PTSD - Military sexual trauma. 2017. Retrieved from http://www. ptsd.va.gov/public/types/violence/military-sexual-trauma-general. asp.

22. Hoy-Ellis CP, Shiu C, Sullivan KM, Kim H-Y, Sturges AM, Fredriksen-Goldsen KI. Prior military service, identity stigma, and mental health among transgender older adults. Gerontologist. 2017;57(S1):S63-71. doi:10.1093/geront/gnw173.

23. Stevens M: Pentagon delays accepting transgender recruits for six months. New York Times June 30, 2017. Retrieved from https:// www.nytimes.com/2017/06/30/us/pentagon-delays-transgenderrecruits.html.

24. BUMEDNOTE 6000 (27 September 2016): Medical treatment of transgender service members - interim guidance. Retrieved from http://www.med.navy.mil/directives/ENotes/Note\%206000\% 20(27\%20Sep\%202016).pdf.

25. American Psychiatric Association: Diagnostic and statistical manual of mental disorders: DSM-5 2013. Arlington, VA: American Psychiatric Association.

26. Hembree WC, Cohen-Kettenis P, Delemarre-van de Waal HA, Gooren LJ, Meyer WJ 3rd, Spack NP, et al. Endocrine treatment of transsexual persons: an Endocrine Society clinical practice guideline. J Clin Endocrinol Metab. 2009;94(9):3132-54.

27. AFPM 2016-36-01 (6 October 2016): In-service transition of airmen identifying as transgender. Retrieved from http://static.epublishing.af.mil/production/1/af_a1/publication/_afpm2016-3601/afpm2016-36-01.pdf.
28. Army Directive 2016-35 (7 October 2016): Army policy on military service of transgender soldiers. Retrieved from https://www. army.mil/e2/c/downloads/453704.pdf.

29. Schvey NA, Blubaugh I, Morettini A, Klein DA. Military family physicians' readiness for treating patients with gender dysphoria. JAMA Intern Med. 2017;13 doi:10.1001/jamainternmed.2017. 0136.

30. Kheel R: VA drops plans to cover surgery for transgender vets. The Hill November 14, 2016. Retrieved from http://thehill.com/policy/ defense/305926-va-drops-plans-to-cover-surgery-for-transgendervets.

31. Kauth MR, Shipherd JC. Transforming a system: improving patient-centered care for sexual and gender minority veterans. LGBT Health. 2016;3(3):1-3. doi:10.1089/lgbt.2016.0047. This paper provides a concise overview of transgender care initiatives in VHA.

32. Shipherd JC, Kauth MR, Matza A. Nationwide interdisciplinary econsultation on transgender care in the Veterans Health Administration. Telemed J E Health. 2016;22(12):1-5. doi:10. 1089/tmj.2016.0013.

33. Kauth MR, Shipherd JC, Lindsay JA, et al. Teleconsultation and training of VHA providers on transgender care: implementation of a multisite hub system. Telemed J E Health. 2015;21:1012-8. doi:10. 1089/tmj.2015.0010.

34. Shipherd JC, Kauth MR, Firek AF, et al. Interdisciplinary transgender veteran care: development of a core curriculum for VHA providers. Transgender Health. 2016;1:54-62. doi:10.1089/tmj.2016. 0013.

35. Kauth MR, Shipherd JC, Barrera TL, Ortigo K, Jones KR. Trainees' perceptions of the VHA interprofessional psychology fellowships in lesbian, gay, bisexual and transgender (LGBT) health. Train Ed Profess Psychol. 2016;10(3):165-70. doi:10. 1037/tep0000123.

36. Sherman M, Kauth MR, Shipherd JC, Street R. Communication between VA providers and sexual and gender minority veterans: a pilot study. Psychol Serv. 2014;11(2):235-42. doi:10.1037/ a0035840.

37. Johnson L, Shipherd JC, Walton H. The psychologist's role in transgender-specific care with U.S. veterans. Psychol Serv. 2016;13(1):69-76. doi:10.1037/ser0000030.

38. U.S. Department of Veterans Affairs, Office of Health Equity: Healthcare Equality Index. Available at www.va.gov/healthequity/ Healthcare_Equality_Index.asp. Accessed March 28, 2016.

39. Human Rights Campaign Foundation. Healthcare Equality Index 2017. Retrieved from http://assets.hrc.org//files/assets/resources/ HEI-2017.pdf?_ga=1.214797335.1064185166.1488312247.

40. Cahill S, Makadon H. Sexual orientation and gender identity data collection in clinical settings and in electronic health records: a key to ending LGBT health disparities. LGBT Health. 2014;1(1):34 41. doi:10.1089/1gbt.2013.0001. This paper highlights the importance of collecting data on sexual orientation and gender identity in electronic health records. 